\title{
Rellenos faciales con ácido hialurónico: Un nuevo desafío en la valoración y predicción de vía área dificultosa
}

\author{
Dermal fillers with hyaluronic acid: A new challenge in the assessment \\ of difficult airway
}

Ivana Sagastume MD. ${ }^{1, *}$

Hospital General de Agudos "Dr. Cosme Argerich".

Fecha de recepción: 02 de agosto de 2021 / Fecha de aceptación: 29 febrero de 2021

\begin{abstract}
Introduction: Difficulties in airway management turn out to be the leading cause of cardiac arrest in anesthesia and they represent $50 \%$ of severe non-fatal anesthesiological complications. The main obstacle lies in the difficulties to predict it. Difficult intubation occurs in 1.5 to $8 \%$ of general anesthesia procedures and it can be defined as one requiring more than three attempts at laryngoscopy or more than 10 minutes of laryngoscopy. In order to identify and prevent an adverse event in patients at risk of presenting a difficult intubation, several parameters have been investigated. Anatomical factors that can predict difficult direct laryngoscopy require a complete pre-anesthetic evaluation and physical examination by the anesthesiologist. These include: the Mallampati scale, the thyromental distance (of Patil), the sternomental distance, the mandibular protrusion test and the atlantooccipital extension; which combined have a high positive predictive value to anticipate difficult airway management.
\end{abstract}

Key words: Tracheal intubation, difficult airway, thyromentonian distance, mallampati.

\section{RESUMEN}

Las dificultades en el abordaje de la vía aérea resultan ser la primera causa de paro cardíaco en anestesia y, a su vez, representan al 50\% de las complicaciones severas no fatales de causa anestesiológica. El principal obstáculo a superar radica en las dificultades para predecirla[1]. La intubación dificultosa se define como la necesidad de tres o más intentos o el empleo de más de 10 minutos para lograr la intubación traqueal, situación que ocurre en 1,5 a 8\% de los procedimientos de anestesia general[1]. Con el fin de identificar pacientes con riesgo de presentar una intubación dificultosa, se han investigado diversos parámetros capaces de anticipar un evento adverso. La caracterización de factores anatómicos que puedan dificultar la laringoscopia directa y la consecuente intubación orotraqueal, requieren de un examen e interrogatorio completo y minucioso por parte del anestesiólogo. Entre estos factores anatómicos encontramos la escala de Mallampati, la distancia tiromentoniana (de Patil)[2],[3] la distancia esternomentoniana, el test de protrusión mandibular y la extensión atlantooccipital; que combinados tienen un alto valor predictivo positivo para anticipar una vía aérea dificultosa[4],[5].

Palabras lave: Vía aérea difícil, rellenos con acido hialurónico.

\section{Introducción}

as dificultades en el abordaje de la vía aérea resultan ser la primera causa de paro cardíaco en anestesia y, a su vez, representan al 50\% de las complicaciones severas no fatales de causa anestesiológica. El principal obstáculo a superar radica en las dificultades para predecirla[1].

La intubación dificultosa se define como la necesidad de tres o más intentos o el empleo de más de 10 minutos para lograr la intubación traqueal, situación que ocurre en 1,5 a 8\% de los procedimientos de anestesia general[1]. Con el fin de identificar pacientes con riesgo de presentar una intubación 
dificultosa se han investigado diversos parámetros capaces de anticipar un evento adverso. La caracterización de factores anatómicos que puedan dificultar la laringoscopia directa y la consecuente intubación orotraqueal, requieren de un examen e interrogatorio completo y minucioso por parte del anestesiólogo. Entre estos factores anatómicos encontramos la escala de Mallampati, la distancia tiromentoniana (de Patil)[2],[3)], la distancia esternomentoniana, el test de protrusión mandibular y la extensión atlantooccipital; que combinados tienen un alto valor predictivo positivo para anticipar una vía aérea dificulto$\mathrm{sa}[4],[5]$.

\section{Caso clínico}

Paciente femenina de 34 años, ASA I, programada para hernioplastía inguinal laparoscópica, sin antecedentes médicos conocidos ni intervenciones quirúrgicas o anestésicas previas. Plan anestésico: anestesia general balanceada con remifentanilo y sevoflurano. En la evaluación preanestésica no se advierten predictores de vía aérea dificultosa, observándose: escala de Mallampati II, distancia tiromentoniana $>6,5 \mathrm{~cm}$, protrusión mandibular grado I, buena flexoextensión cervical.

Tras realizar una preoxigenación durante 3 minutos, se procede a inducción anestésica con fentanilo $2 \mathrm{mcg} / \mathrm{kg}$, propofol $2 \mathrm{mg} / \mathrm{kg}$ y rocuronio $0,6 \mathrm{mg} / \mathrm{kg}$. Ventilación manual satisfactoria. Se efectúa laringoscopía directa y observa un Cormack-Lehane grado 3 que imposibilita la intubación. Se reposiciona a la paciente, se profundiza hipnosis, ventila manualmente y solicita videolaringoscopio McGrath para efectuar segunda maniobra de intubación que resulta satisfactoria.

La intervención quirúrgica transcurre sin complicaciones. Al despertar, en la sala de recuperación, se advierte a la paciente acerca de la dificultad en la intubación orotraqueal y hace hincapié en la importancia de dar a conocer esta información en caso de requerir otra intervención quirúrgica bajo anestesia general. Posteriormente, en un nuevo interrogatorio surge el antecedente de haberse realizado un procedimiento estético en el mentón con ácido hialurónico con el objetivo de mejorar su perfil (Figura 1).

\section{Discusión y Conclusión}

En los últimos años y tras el advenimiento de novedosos tratamientos estéticos de baja complejidad, ambulatorios y realizados en consultorio, se han logrado modificar factores anatómicos que resultan de interés para la evaluación anestésica de la vía aérea y no son referidos en forma espontánea por los pacientes en el interrogatorio, ni pensados dentro de los "procedimientos quirúrgicos y estéticos" capaces de enmascarar una situación de riesgo.

Resultan, principalmente, de interés la proyección del mentón y la perfiloplastía de mandíbula que utilizan al ácido hialurónico como material de relleno, y modifican dramáticamente la anatomía del paciente. Pueden disimular retrognatia o distancia tiromentoniana menor a 6,5-6 cm y, en consecuencia, terminan derivando en una vía aérea dificultosa totalmente inesperada.

A raíz de estos procedimientos, creo que resulta clave profundizar en el interrogatorio durante la evaluación preanes-

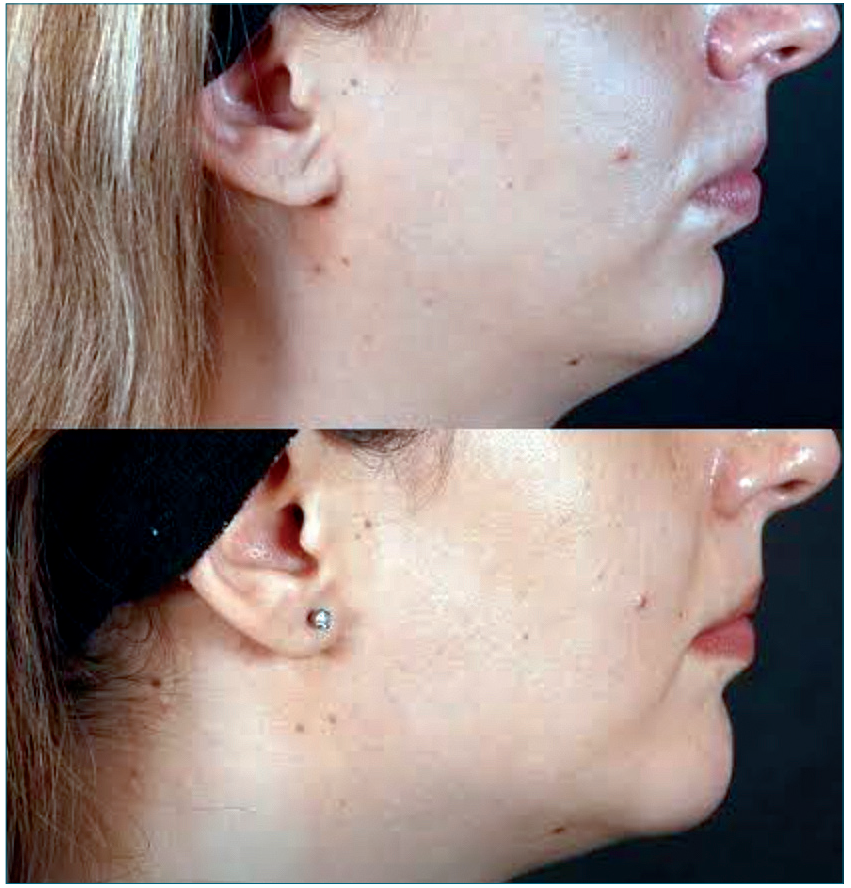

Figura 1. Cambio en el mentón por relleno con ácido hialurónico.

tésica y hacer especial hincapié en este tipo de tratamientos estéticos de fácil accesibilidad con el objetivo de identificar situaciones de riesgo y anticiparse a las complicaciones que pueden ser evitados.

\section{Referencias}

1. $A D$ P. JA W, WB R. Crisis management during anaesthesia: difficult intubation [Internet]. Qual Saf Health Care. 2005;14(3): [cited 2021 Aug 3] Available from: https://pubmed.ncbi.nlm.nih. gov/15933302/

2. Krobbuaban B, Diregpoke S, Kumkeaw S, Tanomsat M. The predictive value of the height ratio and thyromental distance: four predictive tests for difficult laryngoscopy. Anesth Analg. 2005 Nov; 101(5):1542-5. https://doi.org/10.1213/01. ANE.0000181000.43971.1E PMID:16244029

3. Tripathi M, Pandey M; M T. Short thyromental distance: a predictor of difficult intubation or an indicator for small blade selection? [Internet]. Anesthesiology. 2006 Jun;104(6):1131-6. [cited 2021 Aug 3] Available from: https://pubmed.ncbi.nlm.nih. gov/16732082/ https://doi.org/10.1097/00000542-20060600000006 PMID:16732082

4. Orozco-Díaz É, Jorge Álvarez-Ríos J, Luis Arceo-Díaz J, José ..., Ornelas-Aguirre M. Predicción de intubación difícil mediante escalas de valoración de la vía aérea. Cir. 2010;78(5):393 9.

5. Echevarría Hernández AT, Castro YA, Hernández Domínguez K, Díaz Rodríguez C, Sirvent González Y. Pruebas predictivas para la evaluación de la vía aérea en el paciente quirúrgico Prediction tests for airways assessment in surgery patient [Internet]. Rev Cuba Anestesiol Reanim. 2010;9(3):175-85. [cited 2021 Aug 3] Available from: http://scielo.sld.cu 